\title{
Muscular false tendons, aberrant left ventricular papillary musculature, and severe electrocardiographic repolarisation abnormalities:
}

a new syndrome

\author{
Martin G St John Sutton, Simon Dubrey, Paul J Oldershaw
}

\begin{abstract}
Three patients (age 18-39 years; two men) with normal exercise capacity, normal left ventricular function, large muscular false tendons within the left ventricle, and anomalously placed papillary muscles associated with giant $T$ wave inversion on the electrocardiogram are described. The absence of adverse cardiovascular events at long-term follow up (mean 13 years) indicates that this is a benign unrecognised syndrome.
\end{abstract}

(Br Heart f 1994;71:187-190)

Considerable electrocardiographic repolarisation abnormalities noted serendipitously on routine medical employment or insurance examinations in symptom free subjects usually indicate the presence of clinically silent coronary artery disease, left ventricular hypertrophy associated with unrecognised hypertension, hypertrophic cardiomyopathy, or intramyocardial tumour. Their discovery initiates detailed questioning about medical history and careful physical examination. When these show nothing and the cause of the electrocardiographic changes remain unexplained, a sequence of investigations is undertaken that include a chest radiograph, exercise stress test, echocardiogram, and ambulatory electrocardiographic monitoring to ascertain whether the resting abnormalities indicate life threatening arrhythmias. If no cause can be found non-invasively, the coronary arteries are evaluated at cardiac catheterisation.

We present three patients, two of whom were men, with noteably abnormal resting electrocardiograms with $5-15 \mathrm{~mm} T$ wave inversion in at least three electrocardiographic leads. All three patients underwent extensive investigations that included coronary arteriography in the two men. Hypertrophic cardiomypathy was diagnosed in all three patients. This prevented two from obtaining life insurance and substantially altered the third patient's financial situation. The definitive diagnosis in all three patients remained elusive until it was finally established by cross sectional echocardiography after a follow up of 8-18 years (mean 13), during which time none has had any adverse cardiovascular event.

\section{Case reports}

CASE 1

A 48 year old man first sought medical attention at the age of 39 years because of mild atypical chest discomfort unrelated to exertion. He had no risk factors for coronary artery disease and was regularly walking up to 10 miles per week. Physical examination was normal, blood pressure was 110/80 $\mathrm{mm} \mathrm{Hg}$, his chest radiograph was normal, and his electrocardiogram showed sinus rhythm, normal axis, left ventricular hypertrophy with 5-15 mm $T$ wave inversion in the anterolateral leads (I, aVL, and V4-V6) (fig 1). The
Figure 1 Twelve lead electrocardiogram of case 1 showing repolarisation abnormalities in the anterolateral leads I, aVL, V4-V6 with 5-15 mm T wave inversion.

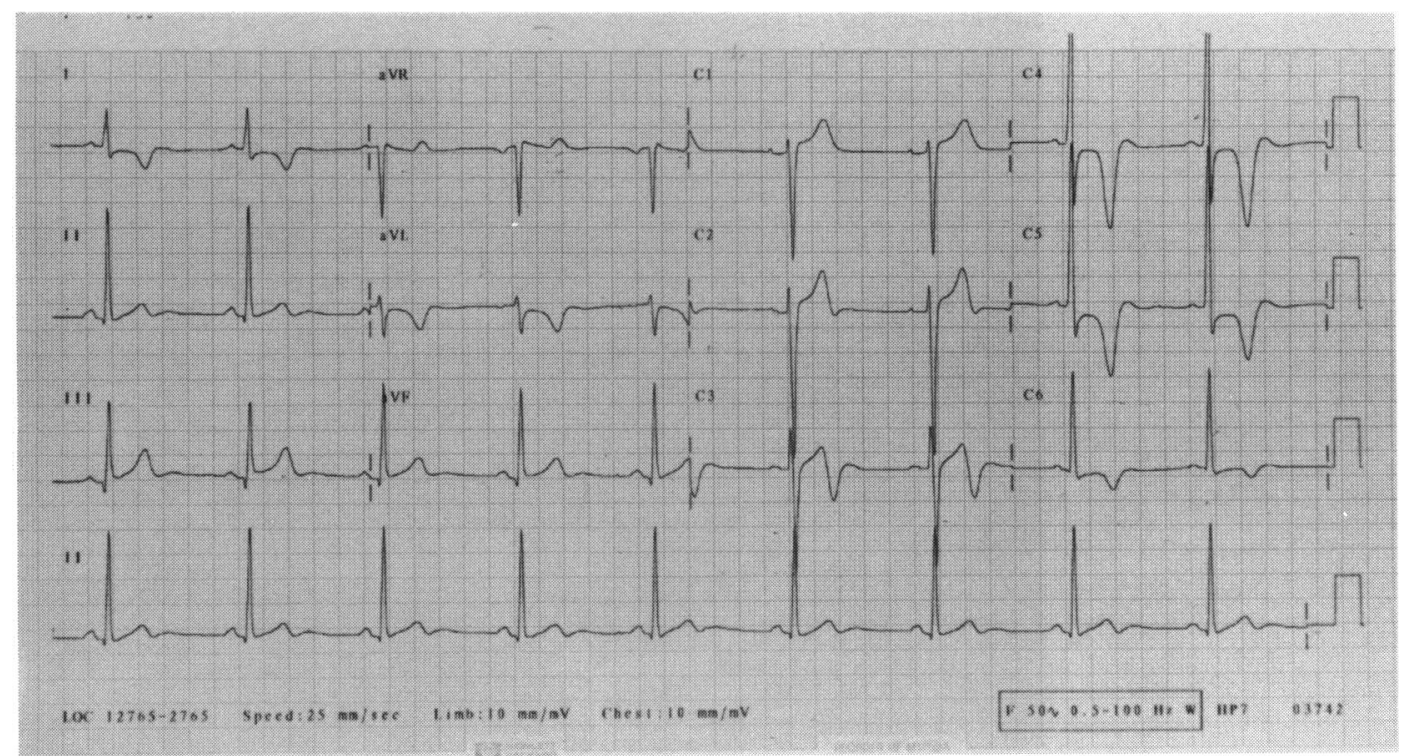


initial echocardiogram showed a normal left ventricular cavity, increased wall thickness at the apex of the left ventricle, normal mitral and aortic valves, no mitral regurgitation, and no intracavity Doppler velocity gradients. He exercised for 14 minutes on a Bruce protocol, stopping only because of leg fatigue, having achieved a peak heart rate of 138 beats/min and a peak blood pressure of $160 / 85 \mathrm{~mm} \mathrm{Hg}$, with 2-3 $\mathrm{mm}$ ST segment depression in electrocardiographic leads (V4-V6). He underwent cardiac catherisation and coronary arteriography that showed entirely normal haemodynamics, no postectopic potentiation of left ventricular systolic pressure or outflow tract pressure gradients, and normal left ventricular function and coronary arteries. He remains symptom free eight years later with an unchanged electrocardiogram. A repeat cross sectional Doppler echocardiogram showed normal left ventricular size, a large muscular false tendon, $1.4 \mathrm{~cm}$ in diameter, originating from the interventricular septum, crossing the left ventricular cavity and bifurcating before inserting partly into the left ventricular apex and partly into the lateral left ventricular free wall (fig 2). Doppler examination of the left ventricular cavity showed no intraventricular velocity gradients. The location of the large muscular trunks and displaced papillary musculature correlated with the location of the persistent electrocardiographic repolarisation abnormalities.

\section{CASE 2}

A 50 year old commercial airline pilot was first noted to have an abnormal resting electrocardiogram with $5 \mathrm{~mm} T$ wave inversion in leads $\mathrm{V} 1$ to $\mathrm{V} 4$ at a routine employment examination at the age of 33 years, at which time he was completely symptom free with a normal exercise tolerance. He had no known risk factors for coronary artery disease. Physical examination was normal, blood pressure was $130 / 80 \mathrm{~mm}$ $\mathrm{Hg}$, and his chest radiograph was normal. The electrocardiogram showed sinus rhythm, normal axis, and the $T$ wave abnormalities were interpreted as anterior myocardial ischaemia. In view of the nature of his job, he underwent a standard exercise stress test. The most recent exercise stress test, performed at the age of 50, was representative of previous tests. He exercised for 12 minutes 15 seconds on a Bruce protocol, stopping because of leg fatigue without chest pain. The peak heart rate achieved was 178 beats $/ \mathrm{min}$ and peak systolic blood pressure was $230 \mathrm{~mm} \mathrm{Hg}$ with 3-4 mm ST segment depression in electrocardiographic leads V2-V6 during exercise at high workload. The initial echocardiogram was reported to show normal left ventricular size with an increased septal thickness of 1.3 $\mathrm{cm}$ and on the basis of this hypertrophic cardiomyopathy was diagnosed. He remained symptom free with no change in the electrocardiogram, and was followed up regularly until the age of 46 years when he underwent cardiac catherisation. Intracardiac pressures, left ventriculography, and coronary arteriography were all entirely normal. During an 11 year follow up, he remains symptom free with no change in the $T$ wave inversion $(5 \mathrm{~mm})$ in electrocardiographic leads V1 to V4. A repeat cross sectional echocardiogram excluded the diagnosis of apical hypertrophic cardiomy-

Figure 2 Echocardiogram of case 1 showing the right ventricle $(R V)$, left ventricle ( $L V$ ), and the large muscular false tendon (FT) taking origin from the proximal

interventricular septum, extending down the length of the cavity, and inserting into the papillary muscle.

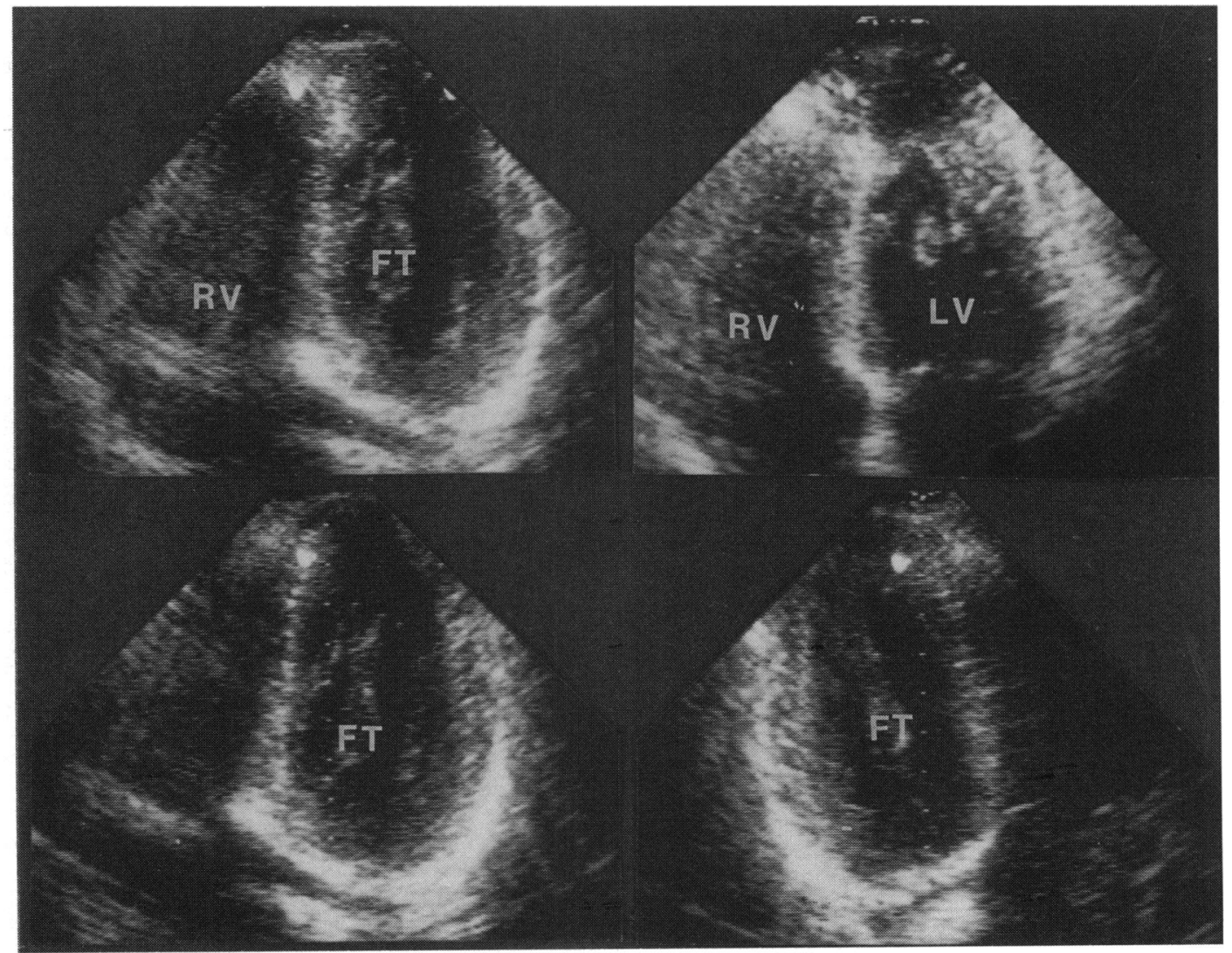


opathy, and showed unequivocally that the septum was of normal thickness. Previous echocardiographic measurements of septal thickness had included a large ectopic muscular false tendon, $5 \mathrm{~mm}$ thick at its origin, that extended from the proximal anterior septum and inserted into two anomalously placed papillary muscles accounting for the apparent asymmetric septal hypertrophy that led to the diagnosis of apical hypertrophic cardiomyopathy. Pulsed, continuous wave, and colour flow Doppler showed that this discrete abnormal muscle bundle crossing the left ventricle did not result in any intracavity obstruction at rest, during, or after isometric exercise. The position of this large abnormal muscle bundle, anomalously located papillary muscles, and the location of the persistent $T$ wave abnormalities in the anterior precordial electrocardiographic leads were concordant.

CASE 3

A 27 year old woman presented at the age of 15 years with palpitation and was found to have an abnormal electrocardiogram that was ascribed to a conduction abnormality. Five years later at a routine employment medical examination, a repeat electrocardiogram showed left ventricular hypertrophy, and an initial echocardiogram was reported to show hypertrophic cardiomyopathy. Aged 23 years she was investigated for paroxysmal dyspnoea and perioral parasthesia that were unrelated to exertion. Physical examination was normal, her blood pressure was $100 / 70 \mathrm{~mm} \mathrm{Hg}$, the electrocardiogram was unchanged with 5-7 $\mathrm{mm} T$ wave inversion in the inferior leads (II,III, and aVF) and $3 \mathrm{~mm} \mathrm{~T}$ wave inversion in the lateral chest leads (V4-V6). An echocardiogram was normal, and in particular showed no features to support the diagnosis of hypertrophic cardiomyopathy. Four years later she had a routine medical check up to obtain life insurance, which was refused on account of her previous diagnosis, even though she was symptom free and swimming an average of 10 miles each week. Twelve years after initial presentation, physical examination remains normal, her blood pressure is $115 / 65 \mathrm{~mm} \mathrm{Hg}$, and her electrocardiogram is unchanged. She exercised for 10 minutes 34 seconds on a Bruce protocol, stopping only because of leg fatigue, having achieved a peak heart rate of 174 beats $/ \mathrm{min}$ and a peak blood pressure of $160 / 70 \mathrm{~mm} \mathrm{Hg}$, with 3-6 mm ST segment depression. A repeat cross sectional echocardiogram showed normal left ventricular chamber size and wall thickness and a large muscular false tendon, $6 \mathrm{~mm}$ in diameter, running from the proximal interventricular septum and inserting both into the inferior surface of the apex of the left ventricle and into the base of the papillary muscles, at which point its diameter was $1.4 \mathrm{~cm}$. There were no echocardiographic signs of apical hypertrophic cardiomyopathy and no intracavity velocity gradients by Doppler. The mitral and aortic valves were normal and there was no mitral regurgitation. The location of the large muscular false tendon crossing the left ventricle and inserting into the inferior and lateral left ventricular wall and the inferolateral location of the electrocardiographic repolarisation abnormalities were similar.

\section{Discussion}

All three patients presented with little in the way of symptoms, maintained normal or above normal exercise capacity, and over a mean follow up period of 13 years none has sustained adverse cardiovascular events. In each of the three patients, clinical attention was focused on the striking repolarisation abnormalities on the resting electrocardiograms. The electrocardiogram became more abnormal during exercise, with additional ST segment depression, which is well known to occur in patients with left ventricular hypertrophy on their resting electrocardiogram. Although clinically silent coronary artery disease is the most likely cause of electrocardiographic repolarisation abnormalities, our patients had no chest pain related to exertion, no family history of any heart disease, no risk factors for coronary artery disease, and each completed in excess of 10.5 minutes of exercise on a Bruce protocol. Before cardiac catheterisation, the working clinical diagnosis in all patients was hypertrophic cardiomyopathy. In two patients $M$ mode echocardiography seemed to substantiate this diagnosis by including the muscular false tendon in the measurements of septal thickness. Cardiac catheterisation was performed in both men for two reasons, firstly to investigate the presence of coronary artery disease even though neither patient had coronary risk factors nor developed symptoms even at high exercise workload, and secondly to either exclude or confirm the diagnosis of hypertrophic cardiomyopathy. Cardiac catheterisation showed normal haemodynamic variables, normal left ventricular function, and excluded both coronary artery disease and obstructive hypertrophic cardiomyopathy, but the cause of the electrocardiographic repolarisation abnormalities remained unknown for several years. The clinical diagnostic label of hypertrophic cardiomyopathy stopped two patients from obtaining life insurance and affected the third patient's ability to earn his living. During follow up from 8-18 years, no patient developed any symptoms or exercise intolerance or had an adverse cardiovascular event, and there was no progression in the electrocardiographic abnormalities. A mean of 13 years after their initial presentations, cross sectional Doppler echocardiograms in each patient showed large muscular trunks known as false tendons within the left ventricle inserting into or displacing the papillary muscles. Repolarisation of these abnormally positioned but normally functioning muscle trunks within the left ventricle very likely accounts for the major $T$ wave inversion on the electrocardiograms. Support for the cause and effect relation between these muscle trunks and the 
electrocardiographic repolarisation abnormalities is provided by the concordance between the location of the muscle trunks and the location of the leads showing notable $T$ wave inversion, and the fact that the electrocardiographic abnormalities were non-progressive at long term follow up.
We conclude that this hitherto unrecognised syndrome of large muscular false tendons within the left ventricle and anomalous papillary musculature associated with giant $T$ wave inversion and normal working capacity is benign and may be easily diagnosed by cross sectional Doppler echocardiography. 